\title{
Upper Bound of the Generalized $p$ Value for the Population Variances of Lognormal Distributions with Known Coefficients of Variation
}

\author{
Rada Somkhuean, Sa-aat Niwitpong, and Suparat Niwitpong \\ Department of Applied Statistics, Faculty of Applied Science, King Mongkut's University of Technology North Bangkok, \\ Bangkok 10800, Thailand \\ Correspondence should be addressed to Sa-aat Niwitpong; sa-aat.n@sci.kmutnb.ac.th
}

Received 27 September 2016; Accepted 15 December 2016; Published 16 January 2017

Academic Editor: Shein-chung Chow

Copyright (C) 2017 Rada Somkhuean et al. This is an open access article distributed under the Creative Commons Attribution License, which permits unrestricted use, distribution, and reproduction in any medium, provided the original work is properly cited.

This paper presents an upper bound for each of the generalized $p$ values for testing the one population variance, the difference between two population variances, and the ratio of population variances for lognormal distribution when coefficients of variation are known. For each of the proposed generalized $p$ values, we derive a closed form expression of the upper bound of the generalized $p$ value. Numerical computations illustrate the theoretical results.

\section{Introduction}

The problem of statistical inference for the population variances has been widely discussed by various authors; see, for example, Singh et al. [1], Agrawal and Sthapit [2], Arcos Cebrián and Rueda García [3], and Arcos et al. [4]. Kadilar and Cingi [5] proposed some ratio estimators for the population variance in simple and stratified random sampling. Cojbasic and Tomovic [6] proposed the bootstrap methods to construct the confidence intervals of the population variance for one sample and the difference of variances of two samples. Cojbasic and Loncar [7] proposed one-sided bootstrap method to construct the confidence intervals of the population variance of skewed distributions. Rajic et al. [8] proposed the new method for the testing one population variance and the difference of variances of two samples, based on $t$-statistics and bootstrap method. Singh and Malik [9] proposed a family of estimators for the population variance using auxiliary attributes. In this paper, we used the generalized $p$ values, proposed by Tsui and Weerahandi [10] and Weerahandi [11], to find new generalized $p$ values for testing: (1) one population variance, (2) the difference between two population variances, and (3) the ratio of population variances of lognormal distributions when coefficients of variation are known. This problem is analogous to the Behrens-Fisher problem; see, for example, Tang and Tsui [12] and Somkhuean et al. [13]. In Section 2, the outline for some basic steps to construct the generalized $p$ value for testing hypothesis in this problem is presented. The process of deriving each of the upper bounds as mentioned above is presented in Section 3. The numerical results are shown in Section 4 and the conclusion is presented in Section 5 .

\section{Generalized $p$ Values}

The concept of the generalized $p$ values has been introduced by Tsui and Weerahandi [10] and Weerahandi [11]. We briefly review this concept as follows.

Let $X$ be a random variable with a density function $f(X \mid$ $\xi)$, where $\xi=(\theta, \delta), \theta$ is the parameter of interest, and $\delta$ is a nuisance parameter.

Suppose we want to test

$$
\begin{array}{r}
H_{0}: \theta \leq \theta_{0} \\
\text { vs. } H_{1}: \theta>\theta_{0} \text {, }
\end{array}
$$


where $\theta_{0}$ is a specified quantity. Let $x$ be a particular observed sample. The generalized test variable, $T(X, x, \xi)$, is required to satisfy the following conditions:

(A1) For fixed $x$ and $\xi=(\theta, \delta)$, the distribution $T(X, x, \xi)$ is free from the nuisance parameter $\delta$.

(A2) $t_{\mathrm{obs}}=T(x, x, \xi)$ is free from any unknown parameter.

(A3) $T(X, x, \xi)$ is either stochastically increasing or decreasing in $\theta$ for any given $t$ and fixed values of $x$ and $\delta$.

Under the above conditions, if $T(X, x, \xi)$ is a stochastically increasing test variable then the subset of space is extreme region $C$. For the one-sided hypothesis given above they defined a data-based extreme region $C_{x}$ is of the form

$$
C_{x}=\{X: T(X, x, \xi) \geq 0\}
$$

Given the observed sample $x$, the generalized $p$ value is defined as

$$
\begin{aligned}
p(x) & =\sup _{\theta \in H_{0}} P(X \in C \mid \theta) \\
& =\sup _{\theta \in H_{0}} P(\{X: T(X, x, \xi) \geq 0\}),
\end{aligned}
$$

for further details and for several applications based on the generalized $p$ value; we refer to the book by Weerahandi [11].

Moreover, Tsui and Weerahandi [10] used the generalized $p$ value $p(x)$ for the Behrens-Fisher problem of testing the difference of two independent normal distribution means with possibly unequal variances. Later, Tang and Tusi [12] extended the works of Weerahandi [11], Gamage and Weerahandi [14] to derive the formula of the upper bound $(r)$ of the generalized $p$ value $p(x)$ which is in the form of (see also, e.g., Kabaila and Lloyd [15])

$$
P(p(x) \leq r) \leq r
$$

In this paper, we also extend Tang and Tsui [12] work to find upper bound of each of the generalized $p$ values, $p(x)$, for hypotheses testing of the one population variance, the difference between two population variances, and the ratio of population variances of lognormal distributions with known coefficients of variation.

\section{Main Results for the Population Variance of Lognormal Distributions with Known Coefficients of Variation}

Let $X_{i}=\left\{X_{i j}, X_{i j}, \ldots, X_{i j}\right\}$, for $\left(i=1,2, j=1,2, \ldots, n_{i}\right)$ be random samples having lognormal distribution and let $Y_{i j}=$ $\ln \left(X_{i j}\right) \sim N\left(\mu_{i}, \sigma_{i}^{2}\right)$ where $\mu_{i}$ and $\sigma_{i}^{2}$ denote the mean and variance of $Y_{i}$, respectively. In particular, the mean, variance, and the coefficient of variation for lognormal distribution are, respectively, given by

$$
\begin{aligned}
E\left(X_{i}\right) & =\exp \left(\mu_{i}+\frac{\sigma_{i}^{2}}{2}\right), \\
\operatorname{var}\left(X_{i}\right) & =\exp \left(2 \mu_{i}+\sigma_{i}^{2}\right)\left(\exp \left(\sigma_{i}^{2}\right)-1\right), \\
\mathrm{CV}_{i} & =\sqrt{\exp \left(\sigma_{i}^{2}\right)-1},
\end{aligned}
$$

where $\mathrm{CV}_{i}$ denotes the coefficient of variation of $X_{i}$ which is computed from $\sqrt{\operatorname{var}\left(X_{i}\right)} / E\left(X_{i}\right)$.

It is easy to see that

$$
\begin{aligned}
\tau_{i} & =\mathrm{CV}=\sqrt{\exp \left(\sigma_{i}^{2}\right)-1}, \\
\tau_{i}^{2} & =\exp \left(\sigma_{i}^{2}\right)-1, \\
\tau_{i}^{2}+1 & =\exp \left(\sigma_{i}^{2}\right), \\
\sigma_{i}^{2} & =\ln \left(\tau_{i}^{2}+1\right)
\end{aligned}
$$

and the parameters of interest are

$$
\begin{aligned}
& \theta_{1}= \operatorname{var}\left(X_{1}\right)=\exp \left(2 \mu_{1}+\ln \left(\tau_{1}^{2}+1\right)\right) \tau_{1}^{2} \\
&= k_{1} \exp \left(2 \mu_{1}+l_{1}\right), \quad k_{1}=\tau_{1}^{2}, l_{1}=\ln \left(\tau_{1}^{2}+1\right), \\
& \theta_{2}= \operatorname{var}\left(X_{1}\right)-\operatorname{var}\left(X_{2}\right) \\
&= \exp \left(2 \mu_{1}+\ln \left(\tau_{1}^{2}+1\right)\right) \tau_{1}^{2} \\
&-\exp \left(2 \mu_{2}+\ln \left(\tau_{2}^{2}+1\right)\right) \tau_{2}^{2} \\
&= k_{1} \exp \left(2 \mu_{1}+l_{1}\right)-k_{2} \exp \left(2 \mu_{2}+l_{2}\right), \\
& k_{i}=\tau_{i}^{2}, l_{i}=\ln \left(\tau_{i}^{2}+1\right), i=1,2, \\
& \theta_{3}= \frac{\operatorname{var}\left(X_{1}\right)}{\operatorname{var}\left(X_{2}\right)}=\frac{k_{1} \exp \left(2 \mu_{1}+l_{1}\right)}{k_{2} \exp \left(2 \mu_{2}+l_{2}\right)} .
\end{aligned}
$$

For testing the null hypothesis, $H_{01}: \theta_{1} \leq \theta_{01}$ vs $H_{a 1}: \theta_{1}>$ $\theta_{01}, H_{02}: \theta_{2} \leq \theta_{02}$ vs $H_{a 2}: \theta_{2}>\theta_{02}$, and $H_{03}: \theta_{3} \leq \theta_{03}$ vs $H_{a 3}: \theta_{3}>\theta_{03}$, the sufficient statistics involving these problems are $\left(\bar{Y}_{i}, S_{i}^{2}\right)$, where

$$
\begin{aligned}
& \bar{Y}_{i}=\frac{1}{n_{i}} \sum_{i=1}^{n_{i}} Y_{i}, \\
& S_{i}^{2}=\frac{1}{n_{i}-1} \sum_{i=1}^{n_{i}}\left(Y_{i j}-\bar{Y}_{i}\right)^{2} .
\end{aligned}
$$

It is known that the probability distributions of the statistics below are independent:

$$
\begin{aligned}
& \bar{Y}_{i} \sim N\left(\mu_{i}, \frac{\sigma_{i}^{2}}{n_{i}}\right), \\
& U_{i}=\frac{\left(n_{i}-1\right) S_{i}^{2}}{\sigma_{i}^{2}} \sim \chi_{n_{i}-1}^{2} .
\end{aligned}
$$


We denote $D=\left\{X_{11}, X_{12}, \ldots, X_{1 n_{1}}\right\}, d=\left\{x_{11}, x_{12}, \ldots, x_{1 n_{1}}\right\}$ and $Q=\left\{X_{11}, X_{12}, \ldots, X_{1 n_{1}}, X_{21}, X_{22}, \ldots, X_{2 n_{2}}\right\}, q=$ $\left\{x_{11}, x_{12}, \ldots, x_{1 n_{1}}, x_{21}, x_{22}, \ldots, x_{2 n_{2}}\right\}$. Here $d$ and $q$ are the vector of the observed samples. Let $\left(\bar{y}_{i}, s_{i}^{2}\right)$ be the observed value of the sufficient statistic $\left(\bar{Y}_{i}, S_{i}^{2}\right)$. Following Tang and Tsui [12] and Somkhuean et al. [13], the repeated sampling, $\left(\bar{y}_{i}, s_{i}^{2}\right)$ follows the same probability distributions as (9).

Case 1. The hypothesis testing is

$$
\begin{gathered}
H_{01}: \theta_{1} \leq \theta_{01}, \\
H_{a}: \theta_{1}>\theta_{01} .
\end{gathered}
$$

The parameter of the one population variance of lognormal distribution when the coefficient of variation is known is

$$
\theta_{1}=k_{1} \exp \left(2 \mu_{1}+l_{1}\right) \text {. }
$$

Using the generalized test variable for $\theta_{1}$ which is

$$
\begin{aligned}
& T\left(X_{1}, x_{1}, \theta_{1}\right)=k_{1} \exp \left(2 \mu_{1}+l_{1}\right) \\
& \quad=k_{1} \exp \left(2\left(\bar{y}_{1}-\frac{\bar{Y}_{1}-\mu_{1}}{S_{1} / \sqrt{n_{1}}} \frac{s_{1}}{\sqrt{n_{1}}}\right)+l_{1}\right) \\
& \quad=k_{1} \exp \left(2\left(\bar{y}_{1}-\frac{Z}{\sqrt{U_{1}} / \sqrt{n_{1}-1}} \frac{s_{1}}{\sqrt{n_{1}}}\right)+l_{1}\right),
\end{aligned}
$$

where $Z=\sqrt{n_{1}\left(\bar{Y}_{1}-\mu_{1}\right)} / \sigma_{1} \sim N(0,1), U_{1}=\left(n_{1}-1\right) S_{1}^{2} / \sigma_{1}^{2} \sim$ $\chi_{n_{1}-1}^{2}$.

It is easy to see that $T\left(X_{1}, x_{1}, \theta_{1}\right)$ in (12) satisfies conditions (A1)-(A3) in Section 2.

The generalized $p$ value, $p(d)$, is defined, under the null hypothesis $H_{01}$, to be

$$
\begin{aligned}
p(d) & =\sup _{H_{01}} P\left(T_{1}\left(X_{1}, x_{1}, \theta_{1}\right) \geq T_{1}\left(x_{1}, x_{1}, \theta_{1}\right)\right) \\
& =P\left(T_{1}\left(X_{1}, x_{1}, \theta_{1}\right) \geq T_{1}\left(x_{1}, x_{1}, \theta_{1}\right)\right) .
\end{aligned}
$$

Following (13) the generalized $p$ value for (10) can be defined as

$$
\begin{aligned}
p(d) & =P\left(T_{1}\left(X_{1}, x_{1}, \theta_{1}\right) \geq T_{1}\left(x_{1}, x_{1}, \theta_{1}\right)\right) \\
& =P\left(k_{1} \exp \left(2\left(\bar{y}_{1}-\frac{Z}{\sqrt{U_{1}} / \sqrt{n_{1}-1}} \frac{s_{1}}{\sqrt{n_{1}}}\right)+l_{1}\right)\right. \\
& \left.\geq \theta_{01}\right)=P\left(\ln \left(k_{1}\right)+2\left(\bar{y}_{1}-\frac{Z}{\sqrt{U_{1}} / \sqrt{n_{1}-1}} \frac{s_{1}}{\sqrt{n_{1}}}\right)+l_{1}\right. \\
& \left.\geq \ln \left(\theta_{01}\right)\right)=P\left(\bar{y}_{1}-\frac{Z}{\sqrt{U_{1}} / \sqrt{n_{1}-1}} \frac{s_{1}}{\sqrt{n_{1}}}\right. \\
& \left.\geq \frac{1}{2}\left(\ln \left(\frac{\theta_{01}}{k_{1}}\right)-l_{1}\right)\right)=P(Z \\
& \left.\leq \frac{\bar{y}_{1}-(1 / 2)\left(\ln \left(\theta_{01} / k_{1}\right)-l_{1}\right)}{s_{1} / \sqrt{n_{1}}}\left(\frac{\sqrt{U_{1}}}{\sqrt{n_{1}-1}}\right)\right) \\
& =E_{U_{1}}\left(\Phi\left(\frac{\bar{y}_{1}-(1 / 2)\left(\ln \left(\theta_{01} / k_{1}\right)-l_{1}\right)}{s_{1} / \sqrt{n_{1}}}\left(\frac{\sqrt{U_{1}}}{\sqrt{n_{1}-1}}\right)\right)\right),
\end{aligned}
$$

where $E_{U_{1}}(\cdot)$ is an expectation operator with respect to $U_{1}=$ $\left(n_{1}-1\right) S^{2} / \sigma^{2} \sim \chi_{n_{1}-1}^{2}$ and $\Phi(\cdot)$ is cdf of the standard normal distribution.

Theorem 1. If $f\left(u_{1}\right)=\Phi\left(T_{n_{1}-1}\left(\sqrt{u_{1}} / \sqrt{n_{1}-1}\right)-m\right)$ then $f\left(u_{1}\right)$ is a convex function of $u_{1}$ where $T_{n_{1}-1}$ is $t$-distribution with $n_{1}-1$ degrees of freedom.

Proof. See Appendix.

Theorem 2. The upper bound of $p(d)$ in (14) takes the form $\Psi_{n_{1}-1}\left(\Phi^{-1}(r)+m\right)$, for $0<r<0.5, m=(1 / 2)\left(\ln \left(\theta_{01} / k_{1}\right)-\right.$ $\left.l_{1}\right) /\left(\sigma_{1} / \sqrt{n}\right)$, where $\Psi(\cdot)$ is a cdf of $t$-distribution function with $\left(n_{1}-1\right)$ degrees of freedom and $\Phi(\cdot)$ is a cdf of the standard normal distribution.

Proof. See Appendix.

Case 2. The hypothesis testing is

$$
\begin{gathered}
H_{02}: \theta_{2} \leq \theta_{02} \\
\text { vs } H_{a 2}: \theta_{2}>\theta_{02} .
\end{gathered}
$$

The parameter of the difference between two population variances for lognormal distributions is

$$
\theta_{2}=k_{1} \exp \left(2 \mu_{1}+l_{1}\right)-k_{2} \exp \left(2 \mu_{2}+l_{2}\right) .
$$

Without loss of generality, suppose $\theta_{2}=\theta_{02}=0$,

$$
\begin{aligned}
\theta_{2} & =k_{1} \exp \left(2 \mu_{1}+l_{1}\right)-k_{2} \exp \left(2 \mu_{2}+l_{2}\right) \\
& =2\left(\mu_{1}-\mu_{2}\right)+\left(\ln \left(\frac{k_{1}}{k_{2}}\right)+\left(l_{1}-l_{2}\right)\right) \\
& =2\left(\mu_{1}-\mu_{2}\right)+D_{1} .
\end{aligned}
$$

Using generalized test variable for $\theta_{2}$ is

$$
\begin{aligned}
& T_{2}\left(X_{1}, X_{2}, x_{1}, x_{2}, \theta_{2}\right)=2\left(\bar{y}_{1}-\bar{y}_{2}\right. \\
& +\frac{\bar{Y}_{2}-\bar{Y}_{1}-\left(\mu_{2}-\mu_{1}\right)}{\sqrt{\sigma_{1}^{2} / n_{1}+\sigma_{2}^{2} / n_{2}}} \sqrt{\left.\frac{\sigma_{1}^{2} s_{1}^{2}}{n_{1} S_{1}^{2}}+\frac{\sigma_{2}^{2} s_{2}^{2}}{n_{2} S_{2}^{2}}\right)+D_{1}} \\
& =2\left(\bar{y}_{1}-\bar{y}_{2}+\frac{Z}{\sqrt{U_{1}+U_{2}}}\right. \\
& \left.\cdot \sqrt{\frac{\left(\left(n_{1}-1\right) / n_{1}\right) s_{1}^{2}}{U_{1} /\left(U_{1}+U_{2}\right)}+\frac{\left(\left(n_{2}-1\right) / n_{2}\right) s_{2}^{2}}{U_{2} /\left(U_{1}+U_{2}\right)}}\right)+D_{1}
\end{aligned}
$$




$$
\begin{aligned}
& =2\left(\bar{y}_{1}-\bar{y}_{2}+\frac{1}{\sqrt{n_{1}+n_{2}-2}}\right. \\
& \cdot \frac{Z}{\sqrt{\left(U_{1}+U_{2}\right) /\left(n_{1}+n_{2}-2\right)}} \\
& \left.\cdot \sqrt{\frac{\left(\left(n_{1}-1\right) / n_{1}\right) s_{1}^{2}}{U_{1} /\left(U_{1}+U_{2}\right)}+\frac{\left(\left(n_{2}-1\right) / n_{2}\right) s_{2}^{2}}{U_{2} /\left(U_{1}+U_{2}\right)}}\right)+D_{1} \\
& =2\left(\bar{y}_{1}-\bar{y}_{2}+\frac{T_{n_{1}+n_{2}-2}}{\sqrt{n_{1}+n_{2}-2}}\right.
\end{aligned}
$$

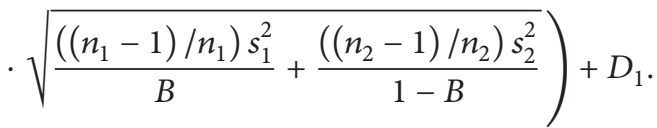

It easy to see that $T_{2}\left(X_{1}, X_{2}, x_{1}, x_{2}, \theta_{2}\right)$ in (18) satisfies conditions (A1)-(A3) in Section 2.

The generalized $p$ value, $p(q)$, is defined, under the null hypothesis $H_{02}$, to be

$$
\begin{aligned}
& p(q)=\sup _{H_{02}} P\left(T_{2}\left(X_{1}, X_{2}, x_{1}, x_{2}, \theta_{2}\right)\right. \\
& \left.\geq T_{2}\left(x_{1}, x_{2}, x_{1}, x_{2}, \theta_{2}\right)\right) \\
& =P\left(T_{2}\left(X_{1}, X_{2}, x_{1}, x_{2}, \theta_{2}\right)\right. \\
& \left.\geq T_{2}\left(x_{1}, x_{2}, x_{1}, x_{2}, \theta_{2}\right)\right)
\end{aligned}
$$

Following (19) the generalized $p$ value for (15) can be defined as

$$
\begin{aligned}
p(q) & =P\left(T_{2}\left(X_{1}, X_{2}, x_{1}, x_{2}, \theta_{2}\right) \geq T_{2}\left(x_{1}, x_{2}, x_{1}, x_{2}, \theta_{2}\right)\right) \\
& =P\left(2 \left(\bar{y}_{1}-\bar{y}_{2}+\frac{T_{n_{1}+n_{2}-2}}{\sqrt{n_{1}+n_{2}-2}}\right.\right. \\
\cdot & \sqrt{\left.\left.\frac{\left(\left(n_{1}-1\right) / n_{1}\right) s_{1}^{2}}{B}+\frac{\left(\left(n_{2}-1\right) / n_{2}\right) s_{2}^{2}}{1-B}\right)+D_{1} \geq 0\right)} \\
& =P\left(\frac{T_{n_{1}+n_{2}-2}}{\sqrt{n_{1}+n_{2}-2}}\right. \\
& \cdot \sqrt{\frac{\left(\left(n_{1}-1\right) / n_{1}\right) s_{1}^{2}}{B}+\frac{\left(\left(n_{2}-1\right) / n_{2}\right) s_{2}^{2}}{1-B} \geq \bar{y}_{2}-\bar{y}_{1}} \\
& -\frac{D_{1}}{2}=P\left(T_{n_{1}+n_{2}-2} \leq\left(\left(\bar{y}_{1}-\bar{y}_{2}\right)+\frac{D_{1}}{2}\right)\right. \\
\cdot & \left.\sqrt{\frac{n_{1}+n_{2}-2}{\left(\left(n_{1}-1\right) / n_{1}\right) s_{1}^{2} / B+\left(\left(n_{2}-1\right) / n_{2}\right) s_{2}^{2} /(1-B)}}\right)
\end{aligned}
$$

$$
\begin{aligned}
& =E_{B}\left(\Psi _ { n _ { 1 } + n _ { 2 } - 2 } \left(\left(\left(\bar{y}_{1}-\bar{y}_{2}\right)+\frac{D_{1}}{2}\right)\right.\right. \\
& \cdot \sqrt{\left.\left.\frac{n_{1}+n_{2}-2}{\left(\left(n_{1}-1\right) / n_{1}\right) s_{1}^{2} / B+\left(\left(n_{2}-1\right) / n_{2}\right) s_{2}^{2} /(1-B)}\right)\right)},
\end{aligned}
$$

where $\Psi_{n_{1}+n_{2}-2}(\cdot)$ is a cdf of $t$-distribution with $n_{1}+n_{2}-2$ degrees of freedom and $E_{B}(\cdot)$ is an expectation operator with respect to $B \cdot\left(B \sim \operatorname{Beta}\left(\left(n_{1}-1\right) / 2,\left(n_{2}-1\right) / 2\right)\right)$.

Theorem 3. If $f(b)=\Psi_{\nu}\left((z+w) \sqrt{1 /\left(k_{1} / b+k_{2} / b\right)}\right)$ then for fixed $z \leq 0$ and $w \geq 0, f(b)$ is convex function for $b$.

Proof. See Appendix.

Theorem 4. If $g(t)=P\left(\Psi_{n_{1}+n_{2}-2}((z+\right.$ $\left.\left.w) \sqrt{1 /\left(t C_{n_{1}-1} /\left(n_{1}-1\right)+(1-t) C_{n_{2}-1} /\left(n_{2}-1\right)\right)}\right) \leq r\right)$ and $z$, $C_{n_{1}-1}, C_{n_{2}-1}$ are independent random variable such that $z \sim N(0,1), C_{n_{1}-1} \sim \chi_{n_{1}-1}^{2}, C_{n_{2}-1} \sim \chi_{n_{2}-1}^{2}$, then $g(t)$ is convex function for $t$.

Proof. See Appendix.

Theorem 5. The upper bound of $p(q)$ is $\Psi_{\min \left\{n_{1}-1, n_{2}-1\right\}}$ $\left(\Psi_{n_{1}+n_{2}-2}^{-1}(r)-w\right)$ for $0<r<0.5, w=\left(D_{1} / 2\right) / \sqrt{\sigma_{1}^{2} / n_{1}+\sigma_{2}^{2} / n_{2}}$, where $\Psi_{n_{1}+n_{2}-2}(\cdot)$ is the $t$-distribution function with $n_{1}+n_{2}-2$ degrees of freedom and $\Psi_{n_{1}+n_{2}-2}^{-1}(\cdot)$ is the inverse function of $\Psi_{n_{1}+n_{2}-2}(\cdot)$.

Proof. See Appendix.

Case 3. The hypothesis testing is

$$
\begin{aligned}
H_{03}: \theta_{3} \leq \theta_{03} \\
\text { vs } H_{a 3}: \theta_{3}>\theta_{03} .
\end{aligned}
$$

The parameter of the ratio of population variances for lognormal distributions is

$$
\theta_{3}=\frac{k_{1} \exp \left(2 \mu_{1}+l_{1}\right)}{k_{2} \exp \left(2 \mu_{2}+l_{2}\right)},
$$

where

$$
\begin{aligned}
& \theta_{3}=\frac{k_{1} \exp \left(2 \mu_{1}+l_{1}\right)}{k_{2} \exp \left(2 \mu_{2}+l_{2}\right)}=\theta_{03} \\
&=\ln \left(\frac{k_{1} \exp \left(2 \mu_{1}+l_{1}\right)}{k_{2} \exp \left(2 \mu_{2}+l_{2}\right)}\right)=\ln \left(\theta_{03}\right) \\
&=2\left(\mu_{1}-\mu_{2}\right)-\left(\ln \left(\frac{k_{2}}{k_{1}}\right)+\left(l_{2}-l_{1}\right)+\ln \theta_{03}\right) \\
&=2\left(\mu_{1}-\mu_{2}\right)-D_{2}=\left(\mu_{1}-\mu_{2}\right)-\frac{D_{2}}{2}, \\
& D_{2}=\ln \left(\frac{k_{2}}{k_{1}}\right)+\left(l_{2}-l_{1}\right)+\ln \theta_{03} .
\end{aligned}
$$


Using generalized test variable for $\theta_{3}$ is

$$
\begin{aligned}
& T_{3}\left(X_{1}, X_{2}, x_{1}, x_{2}, \theta_{3}\right)=\bar{y}_{1}-\bar{y}_{2} \\
& +\frac{\bar{Y}_{2}-\bar{Y}_{1}-\left(\mu_{2}-\mu_{1}\right)}{\sqrt{\sigma_{1}^{2} / n_{1}+\sigma_{2}^{2} / n_{2}}} \sqrt{\frac{\sigma_{1}^{2} s_{1}^{2}}{n_{1} S_{1}^{2}}+\frac{\sigma_{2}^{2} s_{2}^{2}}{n_{2} S_{2}^{2}}}-\frac{D_{2}}{2}=\bar{y}_{1} \\
& -\bar{y}_{2}+Z \sqrt{\frac{\left(n_{1}-1\right) s_{1}^{2}}{n_{1} U_{1}}+\frac{\left(n_{2}-1\right) s_{2}^{2}}{n_{2} U_{2}}}-\frac{D_{2}}{2}=\bar{y}_{1} \\
& -\bar{y}_{2}+\frac{Z}{\sqrt{U_{1}+U_{2}}} \\
& \cdot \sqrt{\frac{\left(\left(n_{1}-1\right) / n_{1}\right) s_{1}^{2}}{U_{1} /\left(U_{1}+U_{2}\right)}+\frac{\left(\left(n_{2}-1\right) / n_{2}\right) s_{2}^{2}}{U_{2} /\left(U_{1}+U_{2}\right)}}-\frac{D_{2}}{2} \\
& =\bar{y}_{1}-\bar{y}_{2}+\frac{1}{\sqrt{n_{1}+n_{2}-2}} \\
& \cdot \frac{Z}{\sqrt{\left(U_{1}+U_{2}\right) /\left(n_{1}+n_{2}-2\right)}} \\
& \cdot \sqrt{\frac{\left(\left(n_{1}-1\right) / n_{1}\right) s_{1}^{2}}{U_{1} /\left(U_{1}+U_{2}\right)}+\frac{\left(\left(n_{2}-1\right) / n_{2}\right) s_{2}^{2}}{U_{2} /\left(U_{1}+U_{2}\right)}}-\frac{D_{2}}{2} \\
& =\bar{y}_{1}-\bar{y}_{2}+\frac{T_{n_{1}+n_{2}-2}}{\sqrt{n_{1}+n_{2}-2}} \\
& \cdot \sqrt{\frac{\left(\left(n_{1}-1\right) / n_{1}\right) s_{1}^{2}}{B}+\frac{\left(\left(n_{2}-1\right) / n_{2}\right) s_{2}^{2}}{1-B}}-\frac{D_{2}}{2} \text {. }
\end{aligned}
$$

It easy to see that $T_{3}\left(X_{1}, X_{2}, x_{1}, x_{2}, \theta_{3}\right)$ in (24) satisfies conditions (A1)-(A3) in Section 2.

The generalized $p$ value, $p\left(q_{2}\right)$, is defined, under the null hypothesis $H_{03}$, to be

$$
\begin{aligned}
& p\left(q_{2}\right)=\sup _{H_{03}} P\left(T_{3}\left(X_{1}, X_{2}, x_{1}, x_{2}, \theta_{3}\right)\right. \\
& \left.\geq T_{3}\left(x_{1}, x_{2}, x_{1}, x_{2}, \theta_{3}\right)\right) \\
& \quad=P\left(T_{3}\left(X_{1}, X_{2}, x_{1}, x_{2}, \theta_{3}\right)\right. \\
& \left.\geq T_{3}\left(x_{1}, x_{2}, x_{1}, x_{2}, \theta_{3}\right)\right) .
\end{aligned}
$$

Following (25) the generalized $p$ value for (21) can be defined as

$$
\begin{aligned}
p\left(q_{2}\right) & =P\left(T_{3}\left(X_{1}, X_{2}, x_{1}, x_{2}, \theta_{3}\right) \geq T_{3}\left(x_{1}, x_{2}, x_{1}, x_{2}, \theta_{3}\right)\right) \\
& =P\left(\bar{y}_{1}-\bar{y}_{2}+\frac{T_{n_{1}+n_{2}-2}}{\sqrt{n_{1}+n_{2}-2}}\right. \\
& \left.\cdot \sqrt{\frac{\left(\left(n_{1}-1\right) / n_{1}\right) s_{1}^{2}}{B}+\frac{\left(\left(n_{2}-1\right) / n_{2}\right) s_{2}^{2}}{1-B}}-\frac{D_{2}}{2} \geq 0\right)
\end{aligned}
$$

$$
\begin{aligned}
& =P\left(\frac{T_{n_{1}+n_{2}-2}}{\sqrt{n_{1}+n_{2}-2}}\right. \\
& \cdot \sqrt{\frac{\left(\left(n_{1}-1\right) / n_{1}\right) s_{1}^{2}}{B}+\frac{\left(\left(n_{2}-1\right) / n_{2}\right) s_{2}^{2}}{1-B} \geq \bar{y}_{2}-\bar{y}_{1}} \\
& \left.+\frac{D_{2}}{2}\right)=P\left(T_{n_{1}+n_{2}-2} \leq\left(\left(\bar{y}_{1}-\bar{y}_{2}\right)-\frac{D_{2}}{2}\right)\right. \\
& \left.\cdot \sqrt{\frac{n_{1}+n_{2}-2}{\left(\left(n_{1}-1\right) / n_{1}\right) s_{1}^{2} / B+\left(\left(n_{2}-1\right) / n_{2}\right) s_{2}^{2} /(1-B)}}\right) \\
& =E_{B}\left(\Psi _ { n _ { 1 } + n _ { 2 } - 2 } \left(\left(\left(\bar{y}_{1}-\bar{y}_{2}\right)-\frac{D_{2}}{2}\right)\right.\right. \\
& \left.\cdot \sqrt{\left.\frac{n_{1}+n_{2}-2}{\left(\left(n_{1}-1\right) / n_{1}\right) s_{1}^{2} / B+\left(\left(n_{2}-1\right) / n_{2}\right) s_{2}^{2} /(1-B)}\right)}\right),
\end{aligned}
$$

where $\Psi(\cdot)$ is a cdf of $t$-distribution with $n_{1}+n_{2}-2$ degrees of freedom and $E(\cdot)$ is an expectation operator with respect to $B \cdot\left(B \sim \operatorname{Beta}\left(n_{1}-1 / 2, n_{2}-1 / 2\right)\right)$.

Theorem 6. The upper bound of $p\left(q_{2}\right)$ is $\Psi_{\min \left\{n_{1}-1, n_{2}-1\right\}}$ $\left(\Psi_{n_{1}+n_{2}-2}^{-1}(r)+w\right)$ for $0<r<0.5, w=\left(D_{2} / 2\right) / \sqrt{\sigma_{1}^{2} / n_{1}+\sigma_{2}^{2} / n_{2}}$, where $\Psi_{n_{1}+n_{2}-2}(\cdot)$ is the $t$-distribution function with $n_{1}+n_{2}-2$ degrees of freedom and $\Psi_{n_{1}+n_{2}-2}^{-1}(\cdot)$ is the inverse function of $\Psi_{n_{1}+n_{2}-2}(\cdot)$.

Proof. It is similar to Theorem 5.

\section{Numerical Results}

In this section, we used functions written in the $\mathbf{R}$ program [16] to compute the values of the upper bounds of the generalized $p$ values proposed in Theorems 2, 5, and 6. For given values of $n_{1}, n_{2}, m, W, \theta_{01}, \theta_{03}$, and $r$, we computed the upper bounds of $p(d), p(q)$, and $p\left(q_{2}\right)$, by using the results from Theorems 2, 5, and 6 shown in Tables 1-3. As we can see in these tables, all results of the upper bounds of the generalized $p$ values proposed in Theorems 2,5 , and 6 depend mainly on a variety of values of $n_{1}, n_{2}, m, W, \theta_{01}, \theta_{03}$, and $r$. As a result, these upper bounds confirm our proof in Theorems 2,5 , and 6 .

\section{Conclusion}

We proposed three new generalized $p$ values for testing the hypotheses of (1) one population variance, (2) the difference between two population variances, and (3) the ratio of population variances of lognormal distributions when the coefficients of variation are known. We also proved new upper bounds for our proposed generalized $p$ values. We note here that the results for these results for case (1), case (2), and case (3) were analogous to the upper bound of the generalized $p$ value for the Behrens-Fisher problem 
TABLE 1: The upper bound of $p$ value for hypothesis (10), for $\tau=1$.

\begin{tabular}{cccccccc}
\hline$n_{1}$ & $\theta_{01}$ & $m$ & $r=0.01$ & $r=0.02$ & $r=0.05$ & $r=0.10$ & $r=0.20$ \\
\hline \multirow{3}{*}{10} & 2.001 & 0.000949333 & 0.02254277 & 0.03514731 & 0.06730417 & 0.1161747 & 0.2111518 \\
& 2.002 & 0.001898192 & 0.02257782 & 0.03520131 & 0.06740329 & 0.1163342 & 0.2114042 \\
& 2.003 & 0.002846578 & 0.02261291 & 0.03525537 & 0.06750250 & 0.1164939 & 0.2116567 \\
\hline \multirow{3}{*}{15} & 2.001 & 0.000949333 & 0.01779592 & 0.02963687 & 0.06122800 & 0.1105716 & 0.2073298 \\
& 2.002 & 0.001898192 & 0.01782805 & 0.02968858 & 0.06132703 & 0.1107338 & 0.2075869 \\
& 2.003 & 0.002846578 & 0.01786022 & 0.02974036 & 0.06142616 & 0.110896 & 0.2078441 \\
\hline \multirow{2}{*}{20} & 2.001 & 0.000949333 & 0.01563906 & 0.02706209 & 0.05832057 & 0.1078731 & 0.2054935 \\
& 2.002 & 0.001898192 & 0.01566962 & 0.02711254 & 0.05841947 & 0.1080364 & 0.2057529 \\
& 2.003 & 0.002846578 & 0.01570022 & 0.02716304 & 0.05851845 & 0.1081999 & 0.2060124 \\
\hline
\end{tabular}

TABLE 2: The upper bound of $p$ value for hypothesis (15), for $\tau_{1}=1, \tau_{2}=1$, and $W=0$.

\begin{tabular}{lccccc}
\hline$n_{1}, n_{2}$ & $r=0.01$ & $r=0.02$ & $r=0.05$ & $r=0.10$ & $r=0.20$ \\
\hline 5,5 & 0.02213745 & 0.03526116 & 0.06823592 & 0.1174918 & 0.2121440 \\
10,10 & 0.01553660 & 0.02705864 & 0.05846833 & 0.1080566 & 0.2055204 \\
15,15 & 0.01356674 & 0.02457767 & 0.05550640 & 0.1052267 & 0.2035670 \\
20,20 & 0.01262788 & 0.02338536 & 0.05407854 & 0.1038673 & 0.2026341 \\
30,30 & 0.01172026 & 0.02222509 & 0.05268530 & 0.1025436 & 0.2017292 \\
\hline
\end{tabular}

TABLE 3: The upper bound of $p$ value for hypothesis (21), for $\tau_{1}=1, \tau_{2}=1$, and $W=0$.

\begin{tabular}{lccccccc}
\hline$n_{1}, n_{2}$ & $\theta_{03}$ & $W$ & $r=0.01$ & $r=0.02$ & $r=0.05$ & $r=0.10$ & $r=0.20$ \\
\hline \multirow{3}{*}{5,5} & 1.01 & 0.00944854 & 0.02234846 & 0.03562201 & 0.06898679 & 0.1188115 & 0.2144116 \\
& 1.02 & 0.01880399 & 0.02255971 & 0.03598345 & 0.06973902 & 0.1201326 & 0.2166765 \\
& 1.03 & 0.02806817 & 0.02277119 & 0.03634547 & 0.07049260 & 0.1214551 & 0.2189384 \\
\hline \multirow{3}{*}{10,10} & 1.01 & 0.00944854 & 0.01577932 & 0.02747895 & 0.05934179 & 0.1095594 & 0.2079965 \\
& 1.02 & 0.01880399 & 0.0160234 & 0.02790139 & 0.06021843 & 0.1110648 & 0.2104685 \\
& 1.03 & 0.02806817 & 0.01626883 & 0.02832593 & 0.06109821 & 0.1125725 & 0.2129362 \\
\hline \multirow{3}{*}{15,15} & 1.01 & 0.00944854 & 0.01381588 & 0.02501398 & 0.05641761 & 0.1067866 & 0.2061060 \\
& 1.02 & 0.01880399 & 0.01406687 & 0.02545308 & 0.05733271 & 0.1083493 & 0.2086403 \\
& 1.03 & 0.02806817 & 0.01431970 & 0.02589493 & 0.05825161 & 0.1099147 & 0.2111700 \\
\hline \multirow{3}{*}{20,20} & 1.01 & 0.009448542 & 0.01287934 & 0.02382890 & 0.05500805 & 0.1054549 & 0.2052033 \\
& 1.02 & 0.01880399 & 0.01313292 & 0.02427558 & 0.05594180 & 0.1070456 & 0.2077678 \\
& 1.03 & 0.02806817 & 0.01338861 & 0.02472537 & 0.05687972 & 0.1086391 & 0.2103272 \\
\hline \multirow{3}{*}{30,30} & 1.01 & 0.009448542 & 0.01197343 & 0.02267532 & 0.05363272 & 0.1041586 & 0.2043280 \\
& 1.02 & 0.01880399 & 0.01222900 & 0.02312906 & 0.05458475 & 0.1057767 & 0.2069217 \\
& 1.03 & 0.02806817 & 0.01248697 & 0.02358628 & 0.05554131 & 0.1073979 & 0.2095102 \\
\hline
\end{tabular}

proposed by Tang and Tsui [12]. Numerical results shown in Tables 1-3 confirmed our results of the upper bounds of the generalized $p$ values proposed in Theorems 2, 5, and 6; we also found that the proposed upper bounds are increasing up on the parameter values of $m$ and $W$. For example, for $n_{1}=10, m=0.002846578$, and $r=0.01$, the upper bound of $p$ value using Theorem 2 is 0.022261291 and this upper bound of $p$ value approaches $r=0.01$ when $n_{1}$ is increasing. Similar results are applied for other cases. For the two-tailed test, that is, $H_{01}: \theta_{1}=\theta_{01}$ and $H_{a}: \theta_{1} \neq \theta_{01}$; it is easy to apply the results of Theorem 2 of Tang and Tsui [12] to all hypotheses in this paper. So we skipped it.

\section{Appendix}

Proof of Theorem 1. Defining $h\left(u_{1}\right)=T_{n_{1}-1}\left(\sqrt{u_{1}} / \sqrt{n_{1}-1}\right)-$ $m$, we have $f\left(u_{1}\right)=\Phi\left(h\left(u_{1}\right)\right)$. Let $\phi$ be the probability density function of $u_{1}$.

Hence

$$
\begin{aligned}
f^{\prime \prime}\left(u_{1}\right)= & \left(f^{\prime}\left(u_{1}\right)\right)^{\prime}=\left(\phi\left(h\left(u_{1}\right)\right) h^{\prime}\left(u_{1}\right)\right)^{\prime} \\
= & \phi^{\prime}\left(h\left(u_{1}\right)\right)\left(h^{\prime}\left(u_{1}\right)\right)^{2} \\
& +\phi\left(h\left(u_{1}\right)\right) h^{\prime \prime}\left(u_{1}\right) .
\end{aligned}
$$

For $T_{n_{1}-1} \leq 0, h\left(u_{1}\right)<0$. Hence $\phi(h(z))>0$ and $\phi^{\prime}(h(z))>0$. 
Moreover

$$
h^{\prime \prime}\left(u_{1}\right)=\left(\frac{1}{2} T_{n_{1}-1} \frac{u_{1}^{-1 / 2}}{\sqrt{n_{1}-1}}\right)^{\prime}=-\frac{1}{4} T_{n_{1}-1} \frac{u_{1}^{-3 / 2}}{\sqrt{n_{1}-1}}
$$$$
>0 \text {. }
$$

Hence $f^{\prime \prime}\left(u_{1}\right)>0$, and $f\left(u_{1}\right)$ is convex in $u_{1}$.

Proof of Theorem 2. Denote $z=\bar{y} /\left(\sigma_{1} / \sqrt{n}\right)$ and $U_{1}=\left(n_{1}-\right.$ 1) $S^{2} / \sigma^{2} \sim \chi_{n_{1}-1}^{2}$.

From (14), we have

$p(d)$

$$
\begin{aligned}
& =E_{U_{1}}\left(\Phi\left(\frac{\bar{y}-(1 / 2)\left(\ln \left(\theta_{01} / k_{1}\right)-l_{1}\right)}{s_{1} / \sqrt{n_{1}}}\left(\frac{\sqrt{U_{1}}}{\sqrt{n_{1}-1}}\right)\right)\right) \\
& =E_{U_{1}}\left(\Phi \left(\left(\frac{\bar{y}}{\sigma_{1} / \sqrt{n_{1}}} \frac{\sqrt{U_{1}}}{\sqrt{n_{1}-1}}\right)\right.\right. \\
& \left.\left.\left.-\frac{(1 / 2)\left(\ln \left(\theta_{01} / k_{1}\right)-l_{1}\right)}{\sigma_{1} / \sqrt{n_{1}}} \frac{\sqrt{U_{1}}}{\sqrt{n_{1}-1}}\right)\left(\frac{\sigma_{1} / \sqrt{n_{1}}}{s_{1} / \sqrt{n_{1}}}\right)\right)\right) \\
& =E_{U_{1}}\left(\Phi \left(\left(z \frac{\sqrt{U_{1}}}{\sqrt{n_{1}-1}}{ }^{(1 / 2)\left(\ln \left(\theta_{01} / k_{1}\right)-l_{1}\right)}\right.\right.\right. \\
& \left.\left.\left.\sigma_{1} / \sqrt{n_{1}} \frac{\sqrt{U_{1}}}{\sqrt{n_{1}-1}}\right)\left(\frac{\sqrt{n_{1}-1}}{\sqrt{U_{1}}}\right)\right)\right) \\
& =E_{U}\left(\Phi\left(T_{n_{1}-1} \frac{\sqrt{U_{1}}}{\sqrt{n_{1}-1}}-m\right)\right), \quad m=\frac{(1 / 2)\left(\ln \left(\theta_{01} / k_{1}\right)-l_{1}\right)}{\sigma_{1} / \sqrt{n_{1}}} .
\end{aligned}
$$

$$
\begin{aligned}
h^{\prime \prime}(b) & =(z+w)\left(\left(\frac{k_{1}}{b}+\frac{k_{2}}{1-b}\right)^{-3 / 2} \cdot\left(-\frac{1}{2}\right)\left(-\frac{k_{1}}{b^{2}}+\frac{k_{2}}{(1-b)^{2}}\right)\right)^{\prime} \\
& =-(z+w)\left(\frac{k_{1}^{2} / 2 b^{2}+k_{2}^{2} / 2(1-b)^{4}+2 k_{1} k_{2} / b(1-b)^{3}+2 k_{1} k_{2} / b^{3}(1-b)+3 k_{1}^{2} k_{2}^{2} / b^{2}(1-b)^{2}}{\left(k_{1} / b+k_{2} /(1-b)\right)^{5 / 2}}\right)>0 .
\end{aligned}
$$

For any $r<0.5$ and $p(d)<r$, hence, by Theorem 1 , we have $E\left(f\left(U_{1}\right)\right) \geq f\left(E\left(U_{1}\right)\right)$

$$
\begin{aligned}
p(d) & =E_{U_{1}}\left(\Phi\left(T_{n_{1}-1} \frac{\sqrt{U_{1}}}{\sqrt{n_{1}-1}}-m\right)\right) \\
& \geq \Phi\left(T_{n_{1}-1} E\left(\frac{\sqrt{U_{1}}}{\sqrt{n_{1}-1}}\right)-m\right) \\
& =\Phi\left(T_{n-1}-m\right)=p_{1}(d) .
\end{aligned}
$$

For $0<r<0.5$, we have

$$
p_{d}(\{d: p(d) \leq r\}) \leq P_{d}\left(\left\{d: p_{1}(d) \leq r\right\}\right)
$$

$$
\begin{aligned}
& =P\left(p_{1}(d) \leq r\right) \\
& =P\left(\Phi\left(T_{n_{1}-1}-m\right) \leq r\right) \\
& =P\left(T_{n_{1}-1} \leq \Phi^{-1}(r)+m\right) \\
& =E\left(\Psi_{n_{1}-1}\left(\Phi^{-1}(r)+m\right)\right) \\
& =\Psi_{n_{1}-1}\left(\Phi^{-1}(r)+m\right),
\end{aligned}
$$

Proof of Theorem 3. Define $f(b)=f(h(b)), h(b)=$ $(z-w) \sqrt{1 /\left(k_{1} / b+k_{2} / b\right)}$, and $\psi_{v}$ is the probability density function of $t$-distribution of $v$ degrees of freedom. Hence

$$
\begin{aligned}
f^{\prime \prime}(h(b)) & =\left(f^{\prime}(h(b))\right)^{\prime}=\left(\psi_{v}(h(b)) h^{\prime}(b)\right)^{\prime} \\
& =\psi_{\nu}^{\prime}(h(b))\left(h^{\prime}(b)\right)^{2}+\phi(h(b)) h^{\prime \prime}(b) .
\end{aligned}
$$

For $z \leq 0, w \geq 0$ implies that $h(b)<0, \psi_{\nu}^{\prime}(h(b)) \geq 0$, and $\psi_{\nu}(h(b)) \geq 0$.

We have
We have $f^{\prime \prime}(h(b))>0$. Hence $f(b)$ is convex function for b.

Proof of Theorem 4.

$$
\begin{aligned}
& \cdot \sqrt{\left.\left.\frac{1}{t C_{n_{1}-1} /\left(n_{1}-1\right)+(1-t) C_{n_{2}-1} /\left(n_{2}-1\right)}\right) \leq r\right)} \\
& =P(((z+w)
\end{aligned}
$$

$$
g(t)=P\left(\Psi_{n_{1}+n_{2}-2}\left((z+w) \quad \cdot \sqrt{\frac{1}{t C_{n_{1}-1} /\left(n_{1}-1\right)+(1-t) C_{n_{2}-1} /\left(n_{2}-1\right)}}\right)\right.
$$




$$
\begin{aligned}
& \left.\leq \Psi_{n_{1}+n_{2}-2}^{-1}(r)\right)=P(z \\
& \left.\leq \sqrt{\frac{t C_{n_{1}-1}}{n_{1}-1}+\frac{(1-t) C_{n_{2}-1}}{n_{2}-1}}\left(\Psi_{n_{1}+n_{2}-2}^{-1}(r)\right)-w\right) \\
& =E\left(\Phi \left(\sqrt{\frac{t C_{n_{1}-1}+\frac{(1-t) C_{n_{2}-1}}{n_{1}-1}}{n_{2}-1}}\left(\Psi_{n_{1}+n_{2}-2}^{-1}(r)\right)\right.\right. \\
& -w))
\end{aligned}
$$

$$
\begin{aligned}
& \cdot\left(\frac{t C_{n_{1}-1}}{n_{1}-1}+\frac{(1-t) C_{n_{2}-1}}{n_{2}-1}\right)^{-1 / 2} \\
& \left.\cdot\left(\frac{C_{n_{1}-1}}{n_{1}-1}-\frac{C_{n_{2}-1}}{n_{2}-1}\right)\right)^{\prime}=\left(-\frac{1}{4} \Psi_{n_{1}+n_{2}-2}^{-1}(r)\right. \\
& \cdot\left(\frac{t C_{n_{1}-1}}{n_{1}-1}+\frac{(1-t) C_{n_{2}-1}}{n_{2}-1}\right)^{-3 / 2} \\
& \left.\cdot\left(\frac{C_{n_{1}-1}}{n_{1}-1}-\frac{C_{n_{2}-1}}{n_{2}-1}\right)^{2}\right)^{\prime} \geq 0 .
\end{aligned}
$$

where $\Phi$ is the cdf of a standard normal distribution.

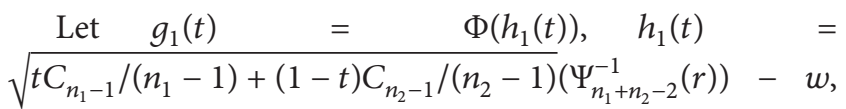
and $\phi$ is the probability density function of a standard normal distribution. Hence

$$
\begin{aligned}
g_{1}^{\prime \prime}(t) & =\left(g_{1}^{\prime}(t)\right)^{\prime}=\left(\phi\left(h_{1}(t)\right) h_{1}^{\prime}(t)\right)^{\prime} \\
& =\phi^{\prime}\left(h_{1}(t)\right)\left(h_{1}^{\prime}(t)\right)^{2}+\phi\left(h_{1}(t)\right) h_{1}^{\prime \prime}(t) .
\end{aligned}
$$

We have

$$
h_{1}^{\prime \prime}(t)=\left(\frac{1}{2} \Psi_{n_{1}+n_{2}-2}^{-1}(r)\right.
$$

(A.10)

We have $g_{1}^{\prime \prime}(t) \geq 0$. Hence $g_{1}(t)$ is convex function for $t$. As a result, $g(t)=E\left(g_{1}(t)\right)$ is convex in $t$.

Proof of Theorem 5. Denote

$$
\begin{aligned}
T & =\frac{\sigma_{1}^{2} / n_{1}}{\sigma_{1}^{2} / n_{1}+\sigma_{2}^{2} / n_{2}}, \\
z & =\frac{\left(\bar{y}_{1}-\bar{y}_{2}\right)}{\sqrt{\sigma_{1}^{2} / n_{1}+\sigma_{2}^{2} / n_{2}}}, \\
C_{n_{1}-1} & =\frac{\left(n_{1}-1\right) s_{1}^{2}}{\sigma_{1}^{2}}, \\
C_{n_{2}-1} & =\frac{\left(n_{2}-1\right) s_{2}^{2}}{\sigma_{2}^{2}} .
\end{aligned}
$$

From (24)

$$
\begin{aligned}
& p(q) \\
& =E_{B}\left(\Psi_{n_{1}+n_{2}-2}\left(\frac{\left(\bar{y}_{1}-\bar{y}_{2}\right)+D_{1} / 2}{\sqrt{\sigma_{1}^{2} / n_{1}+\sigma_{2}^{2} / n_{2}}} \sqrt{\left.\frac{n_{1}+n_{2}-2}{\left(\left(n_{1}-1\right) / n_{1}\right) s_{1}^{2} / B+\left(\left(n_{2}-1\right) / n_{2}\right) s_{2}^{2} /(1-B)}\left(\frac{1}{1 /\left(\sigma_{1}^{2} / n_{1}+\sigma_{2}^{2} / n_{2}\right)}\right)\right)}\right),\right. \\
& =E_{B}\left(\Psi_{n_{1}+n_{2}-2}\left((z+w) \sqrt{\left.\frac{n_{1}+n_{2}-2}{\left(C_{n_{1}-1} \sigma_{1}^{2} / n_{1}\right) / B\left(\sigma_{1}^{2} / n_{1}+\sigma_{2}^{2} / n_{2}\right)+\left(C_{n_{2}-1} \sigma_{2}^{2} / n_{2}\right) /(1-B)}\right)}\right), \frac{D_{1} / 2}{\sqrt{\sigma_{1}^{2} / n_{1}+\sigma_{2}^{2} / n_{2}}}\right. \\
& =E_{B}\left(\Psi_{n_{1}+n_{2}-2}\left((z+w) \sqrt{\frac{n_{1}+n_{2}-2}{T C_{n_{1}-1} / B+(1-T) C_{n_{2}-1} /(1-B)}}\right)\right) .
\end{aligned}
$$

For any $r<0.5$ and $p(q)<r$, hence, by Theorem 1 , such that $E_{B}(f(B)) \geq f(E(B))$

$$
\begin{aligned}
& \left.\cdot \sqrt{\left.\frac{n_{1}+n_{2}-2}{T C_{n_{1}-1} / B+(1-T) C_{n_{2}-1} /(1-B)}\right)}\right) \\
& \geq \Psi_{n_{1}+n_{2}-2}\left(E_{B}((z+w)\right.
\end{aligned}
$$

$$
p(q)=E_{B}\left(\Psi_{n_{1}+n_{2}-2}((z+w)\right.
$$




$$
\begin{aligned}
& \cdot \sqrt{\left.\left.\frac{n_{1}+n_{2}-2}{T C_{n_{1}-1} / B+(1-T) C_{n_{2}-1} /(1-B)}\right)\right)} \\
& =\Psi_{n_{1}+n_{2}-2}((z+w) \\
& \cdot \sqrt{\left.\frac{1}{T C_{n_{1}-1} /\left(n_{1}-1\right)+(1-T) C_{n_{2}-1} /\left(n_{2}-1\right)}\right)} \\
& =p_{1}(q) .
\end{aligned}
$$

For $0<r<0.5$, we have

$$
\begin{aligned}
& p_{q}(\{q: p(q) \leq r\}) \leq P_{q}\left(\left\{q: q_{1}(q) \leq r\right\}\right)=g(T), \\
& \text { by Theorem } 4 \text {, } \\
& g(T) \leq \max \{g(0), g(1)\} \\
& =\max \left\{P\left(\Psi_{n_{1}+n_{2}-2}\left((z+w) \sqrt{\frac{1}{C_{n_{1}-1} /\left(n_{1}-1\right)}}\right) \leq r\right),\right. \\
& \left.P\left(\Psi_{n_{1}+n_{2}-2}\left((z+w) \sqrt{\frac{1}{C_{n_{2}-1} /\left(n_{2}-1\right)}}\right) \leq r\right)\right\} \\
& =\max \left\{P \left(z \sqrt{\frac{1}{C_{n_{1}-1} /\left(n_{1}-1\right)}} \leq-w \sqrt{\frac{1}{C_{n_{1}-1} /\left(n_{1}-1\right)}}\right.\right. \\
& \left.+\Psi_{n_{1}+n_{2}-2}^{-1}(r)\right), P\left(z \sqrt{\frac{1}{C_{n_{2}-1} /\left(n_{2}-1\right)}}\right. \\
& \left.\left.\leq-w \sqrt{\frac{1}{C_{n_{2}-1} /\left(n_{2}-1\right)}}+\Psi_{n_{1}+n_{2}-2}^{-1}(r)\right)\right\} \\
& =\max \left\{E\left(\Psi_{n_{1}-1}\left(-w \sqrt{\frac{1}{C_{n_{1}-1} /\left(n_{1}-1\right)}}+\Psi_{n_{1}+n_{2}-2}^{-1}(r)\right)\right),\right. \\
& \left.E\left(\Psi_{n_{2}-1}\left(-w \sqrt{\frac{1}{C_{n_{2}-1} /\left(n_{2}-1\right)}}+\Psi_{n_{1}+n_{2}-2}^{-1}(r)\right)\right)\right\} \\
& \geq \max \left\{\Psi_{n_{1}-1}\left(E\left(-w \sqrt{\frac{1}{C_{n_{1}-1} /\left(n_{1}-1\right)}}\right)+\Psi_{n_{1}+n_{2}-2}^{-1}(r)\right),\right. \\
& \left.\Psi_{n_{2}-1}\left(E\left(-w \sqrt{\frac{1}{C_{n_{2}-1} /\left(n_{2}-1\right)}}\right)+\Psi_{n_{1}+n_{2}-2}^{-1}(r)\right)\right\} \\
& =\Psi_{\min \left\{n_{1}-1, n_{2}-1\right\}}\left(\Psi_{n_{1}+n_{2}-2}^{-1}(r)-w\right), \quad w=\frac{D_{1} / 2}{\sqrt{\sigma_{1}^{2} / n_{1}+\sigma_{2}^{2} / n_{2}}} .
\end{aligned}
$$

\section{Competing Interests}

The authors declare that there is no conflict of interests regarding the publication of this paper.

\section{Acknowledgments}

The second author is grateful to Grant no. KMUTNB-GOV59-36 from King Mongkut's University of Technology North Bangkok.

\section{References}

[1] H. P. Singh, L. N. Upadhyaya, and U. D. Namjoshi, "Estimation of finite population variance," Current Science, vol. 57, no. 24, pp. 1331-1334, 1988.

[2] M. C. Agrawal and A. B. Sthapit, "Unbiased ratio-type variance estimation," Statistics and Probability Letters, vol. 25, no. 4, pp. 361-364, 1995.

[3] A. Arcos Cebrián and M. Rueda García, "Variance estimation using auxiliary information: an almost unbiased multivariate ratio estimator," Metrika. International Journal for Theoretical and Applied Statistics, vol. 45, no. 2, pp. 171-178, 1997.

[4] A. Arcos, M. Rueda, M. D. Martínez, S. González, and Y. Román, "Incorporating the auxiliary information available in variance estimation," Applied Mathematics and Computation, vol. 160, no. 2, pp. 387-399, 2005.

[5] C. Kadilar and H. Cingi, "Ratio estimators for the population variance in simple and stratified random sampling," Applied Mathematics and Computation, vol. 173, no. 2, pp. 1047-1059, 2006.

[6] V. Cojbasic and A. Tomovic, "Nonparametric confidence intervals for population variance of one sample and the difference of variances of two samples," Computational Statistics \& Data Analysis, vol. 51, no. 12, pp. 5562-5578, 2007.

[7] V. Cojbasic and D. Loncar, "One-sided confidence intervals for population variances of skewed distributions," Journal of Statistical Planning and Inference, vol. 141, no. 5, pp. 1667-1672, 2011.

[8] V. C. Rajic, J. Kocovic, D. Loncar, and T. R. Antic, “Testing population variance in case of one sample and the difference of variances in case of two samples: example of wage and pension data sets in Serbia," Economic Modelling, vol. 29, no. 3, pp. 610613, 2012.

[9] R. Singh and S. Malik, "Improved estimation of population variance using information on auxiliary attribute in simple random sampling," Applied Mathematics and Computation, vol. 235, pp. 43-49, 2014.

[10] K.-W. Tsui and S. Weerahandi, "Generalized $p$-values in significance testing of hypotheses in the presence of nuisance parameters," Journal of the American Statistical Association, vol. 84, no. 406, pp. 602-607, 1989.

[11] S. Weerahandi, Exact Statistical Methods for Data Analysis, Springer, New York, NY, USA, 1995.

[12] S. Tang and K.-W. Tsui, "Distributional properties for the generalized $p$-value for the Behrens-Fisher problem," Statistics \& Probability Letters, vol. 77, no. 1, pp. 1-8, 2007.

[13] R. Somkhuean, S. Niwitpong, and S.-A. Niwitpong, "Upper bounds of generalized p-values for testing the coefficients of variation of lognormal distributions," Chiang Mai Journal of Science, vol. 43, pp. 671-681, 2016.

[14] J. Gamage and S. Weerahandi, "Size performance of some tests in one-way ANOVA," Communications in Statistics-Simulation and Computation, vol. 27, no. 3, pp. 625-640, 1998.

[15] P. Kabaila and C. J. Lloyd, "Tight upper confidence limits from discrete data," The Australian Journal of Statistics, vol. 39, no. 2, pp. 193-204, 1997.

[16] The $\mathrm{R}$ Development Core Team, An Introduction to $R, \mathrm{R}$ Foundation for Statistical Computing, Vienna, Austria, 2010, http://www.R-project.org. 


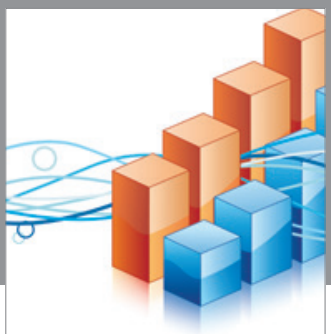

Advances in

Operations Research

vatem alat4

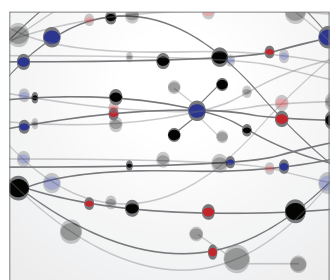

\section{The Scientific} World Journal
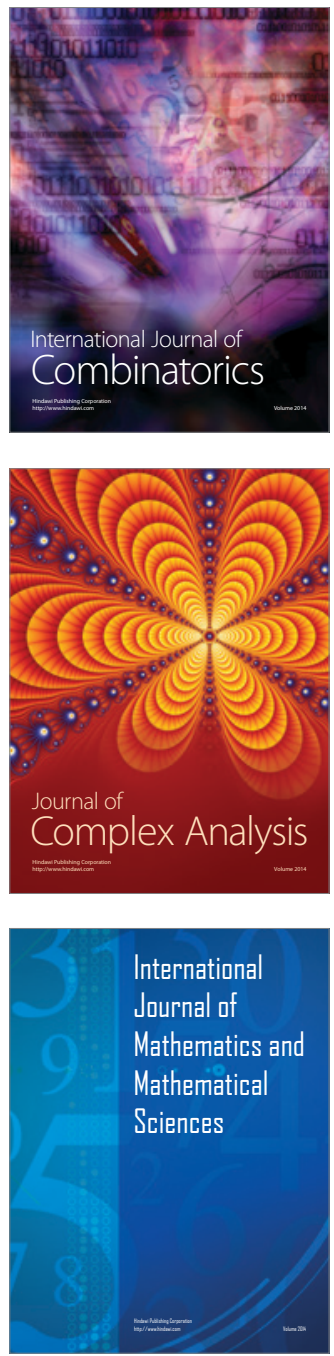
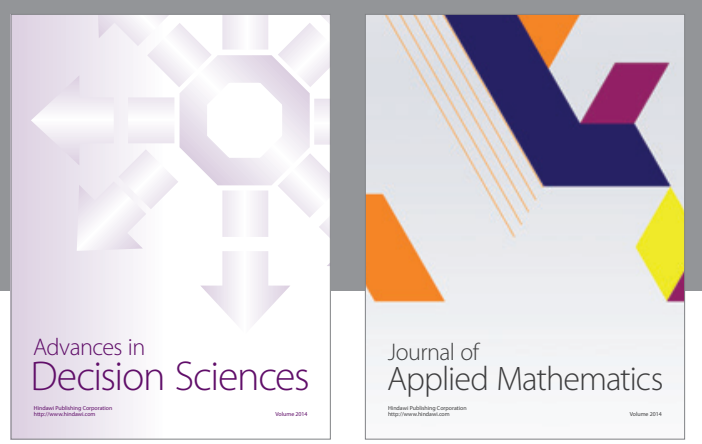

Algebra

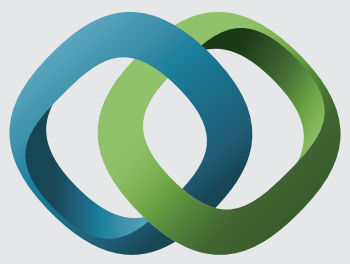

\section{Hindawi}

Submit your manuscripts at

https://www.hindawi.com
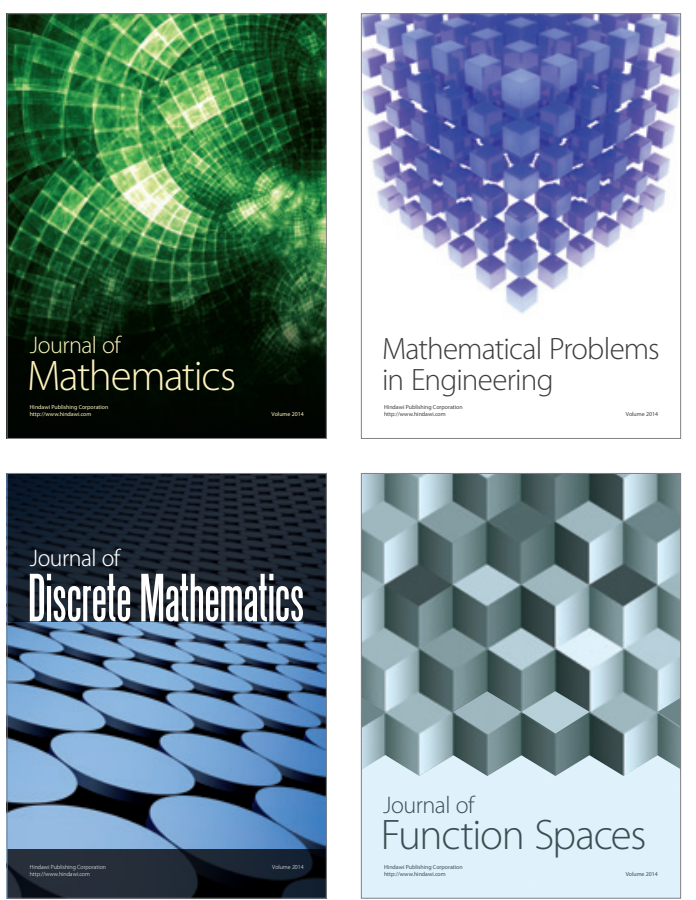

Mathematical Problems in Engineering
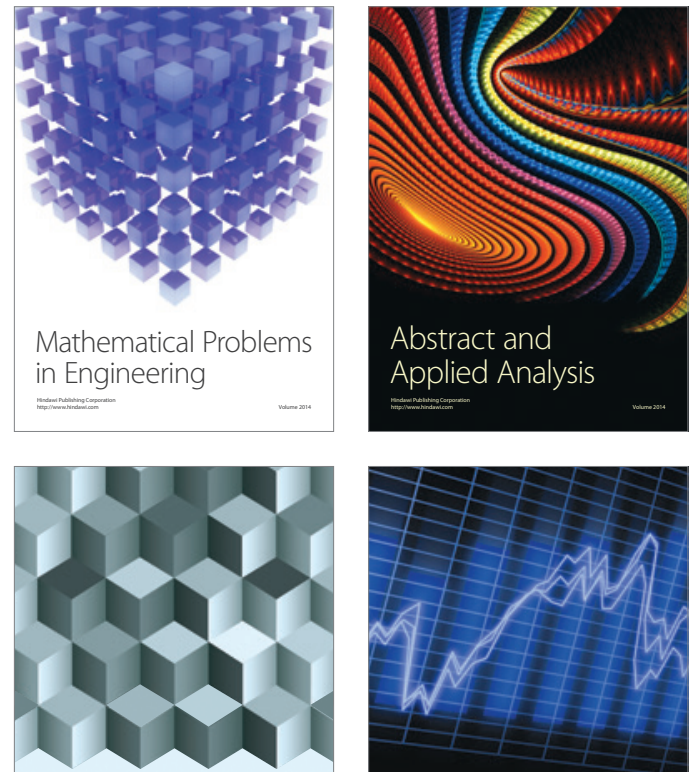

Journal of

Function Spaces

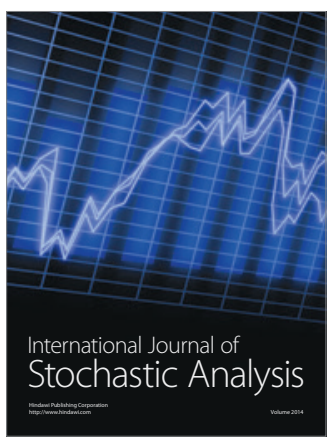

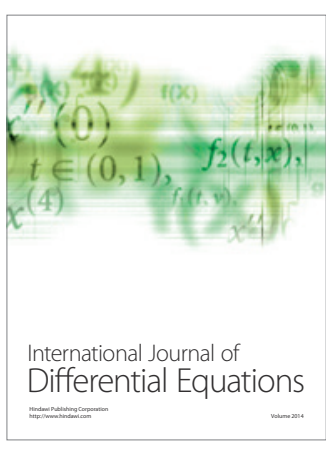
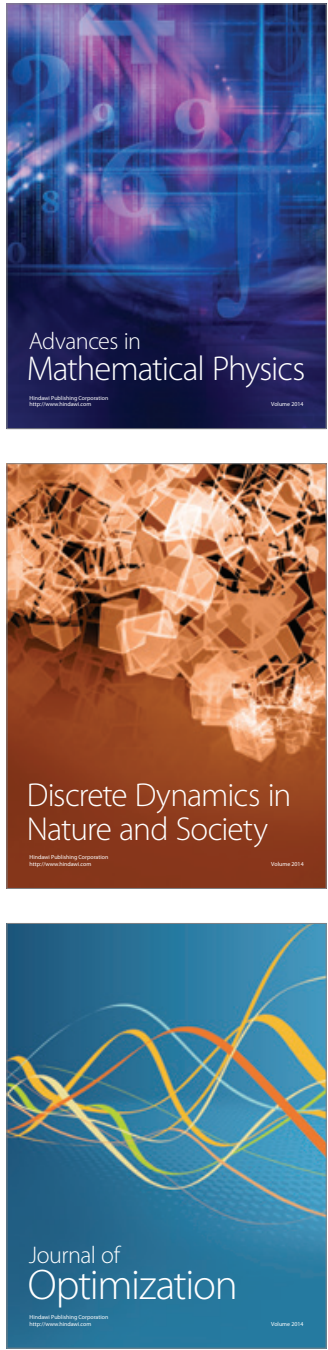